\title{
Use of an Artificial Neural Network-based Metamodel to Reduce the Computational Cost in a Ray-tracing Prediction Model
}

\author{
Sheila S. Travessa, Walter P. Carpes Jr., \\ Federal University of Santa Catarina, Electric Engineering Department \\ Grupo de Concepção e Análise de Dispositivos Eletromagnéticos (GRUCAD) \\ Florianópolis, Brazil \\ sheila.santisi@gmail.com,walterpcjr@gmail.com
}

\begin{abstract}
The purpose of this article is based on analyzing the use of RTQ3D ("quasi-3D" ray tracing technique) to produce the value of the initial electromagnetic fields or fitness for a hundred and sixty receivers according to the possible positions of two antennas to be distributed in a closed environment. The problem variables consist of the values of the magnetic fields for one hundred and sixty receptors depending on the positions of the antennas to the base stations, which serve as input data for the algorithm to the RMLP (Artificial Neural Network, multilayer perceptron with Real backpropagation learning algorithm). The values of the magnetic fields associated with the positions of the antennas are the values to be learned by the network, the teacher of RMLP. This study aims to develop efficient techniques for optimization of electromagnetic problems. We use the PSO (Particle Swarm Optimization) algorithm associated with a metamodel based on an ANN (Artificial Neural Network). Specifically, we use the MLP (Multilayer Perceptron) with the backpropagation algorithm in order to evaluate objective functions in an efficient way. The ANN will be used to assist the technique of "quasi 3D" ray-tracing in order to reduce the high computational cost of this technique in PSO optimization.
\end{abstract}

Index Terms- Artificial Neural Networks, Multilayer Perceptron, electromagnetic fields, Particle Swarm Optimization, metamodeling.

\section{INTRODUCTION}

Optimization problems are characterized by situations in which one needs to maximize or minimize a numerical function of several variables, in a context where there may be restrictions.

The sophistication of computer resources developed in recent years has motivated a breakthrough in optimization techniques. In fact, optimization problems have become increasingly complex, increasing the associated computational cost. Classical optimization techniques are reliable and have applications in many different fields of engineering and other sciences. However, deterministic methods can present some numerical difficulties and problems related to robustness: the lack of continuity of the functions to be optimized or its constraints, nonconvex functions, multimodality, the existence of noise functions, the need to work with discrete values for variables, the existence of local minimum or maximum, etc. Thus, studies of heuristic methods with search-controlled randomized 
probabilistic criteria reappear as a strong trend in recent years, mainly due to their robustness and to the advancement of computational resources. One of the main motivations for using stochastic optimization methods, such as PSO, is their ability to converge to the global optimum point. Indeed, most of the electromagnetics problems are multimodal, and using a deterministic method could lead to a local optimum. However, a limiting factor of stochastic methods is the need for a large number of objective function evaluations [1].

In the methods of natural optimization, the objective function is evaluated several times, since we deal with a great number of points (population) at a given iteration. This increases the computational cost associated with these methods. However, this high computational cost is the price to pay for preventing solutions from becoming trapped in local minima.

In general, the methods of natural optimization require more computational effort when compared to classical methods, but have some advantages such as ease of implementation, robustness and do not require continuity of the objective function associated with the problem [2].

An important application of metamodels is in the optimization of electromagnetic problems. In fact, given that the modeling of such problems generally has a high computational cost, the optimization of electromagnetic devices often requires certain procedures for the replacement of objective functions, in order to evaluate them in an efficient way. The replacement function allows obtaining results with good precision, but with a much lower computational cost [3].

Several models can be used to obtain approximations of objective functions with good accuracy. The most popular are the polynomial model, Kriging model, feedforward neural networks, including multilayer perceptrons, radial basis function networks and support vector machines. According to [4], the development of strategies for assisted metamodels are proposed as a new approach to basic simulation optimization in the field of electromagnetic project. In this application field, the estimation of objective functions are likely to have a significant computational cost, and techniques such as metamodeling are applied to accelerate the evolutionary optimization strategies. Some experiments done with the main metamodels mentioned in this study showed that better results were obtained with the Kriging model and artificial neural network.

The Kriging model [3] and the feed-forward neural network are robust and offer savings in computational time. Studies have shown that these two metamodels have similar performance [5]. Therefore, we chose to use the Artificial Neural Networks (ANN), which have been widely used in several applications due to ease of implementation and effectiveness.

It is important to stress that the evolutionary algorithms associated with metamodels are becoming extensively used by a growing number of researchers in design optimization [6]. Here, we show the validity of the PSO (Particle Swarm Optimization) algorithm in conjunction with an ANN-based metamodel to be used in the optimization of electromagnetic problems. 


\section{PSO, RTQ3D AND THE ANN-BASED METAMODEL}

\section{A. PSO - Particle Swarm Optimization}

Introduced by Kennedy and Eberhart in 1995, the particle swarm optimization has emerged from experiments with algorithms modeled from the observation of social behavior of certain types of birds. The particles considered by the algorithm behave like birds looking for food or a place to make their nests, each one using both its own knowledge and the knowledge of the flock (or swarm) concerned to the best location for that. PSO is composed of particles represented by vectors defining the current speed of each particle and its location. The location of each particle is updated according to its current speed, its own knowledge and the knowledge acquired by the whole flock. The PSO algorithm encompasses simple concepts and can be implemented in a few lines of code, requiring only simple mathematical operators.

The algorithm starts by generating a population of $\mathrm{N}$ particles that form the swarm and establish (randomly) their respective positions in the search space. It also sets the initial speed for each particle. Subsequently, at each iteration $k$, the algorithm updates the position $x^{i}(k)$ and the velocity $v^{i}(k)$ of each particle $i$. After the first evaluation of the fitness associated to a given particle, the algorithm keeps track of the coordinates $p^{i}$ in the search space associated with its best solution. The overall best value (considering the whole population) and its location $p^{s}(k)$ are also stored during the procedure. With a simple scheme, these stored data are iteratively updated using random parameters and constants, changing the particles velocities in order to intelligently explore the search domain at each iteration [7].

The velocity vector of each particle is updated according to (1):

$$
v^{i}(k+1)=w v^{i}(k)+c_{1} r_{1}\left[p^{i}-x^{i}(k)\right]+c_{2} r_{2}\left[p^{s}(k)-x^{i}(k)\right]
$$

Where $w$ is the inertia weight; $\mathrm{c}_{1}$ and $\mathrm{c}_{2}$ are, respectively, the cognition and social coefficient; and $\mathrm{r}_{1}$ and $\mathrm{r}_{2}$ are random numbers uniformly distributed in $[0,1]$. Inertia weight $w$ usually is chosen between 0.4 and 1.4: lower values speed up convergence while higher values encourage exploring the search space. The value used for $w$ in this study was 0.9 , which allows obtaining very good results for the analyzed problem. Cognition coefficient $c_{1}$ limits the size of the step that the particle takes toward its individual best while social coefficient $c_{2}$ limits the size of the step that the particle takes toward the global best. Usually both assume values close to 2 . It would be possible to use different values of $\mathrm{c}_{1}$, $c_{2}$ and $w$, including the idea of using variable parameters in time (e.g., decreasing $w$ with the advancement of iterations). This could have been done to improve the convergence. However, we use typical fixed values for these parameters, according to the literature, which also yields satisfactory results.

The position of each particle is updated according to (2):

$$
x^{i}(k+1)=x^{i}(k)+v^{i}(k+1)
$$

The iterations proceed until some stopping criterion is reached (e.g., maximum number of iterations or desired particle fitness). 


\section{B. An RTQ3D-indoor}

For the purpose presented in this paper, the RTQ3D-indoor algorithm is used for field computation and coverage evaluation, since the applications are in indoor environments. The RTQ3D-indoor [8] model uses 2D ray-tracing algorithm based on the Theory of Images to build a "tree of images" and to find the different reflected rays for a given scenario. The input scenario database is described in the horizontal plane, but adding the ceiling height from the floor and the transmitting (base stations) and receiving (user terminals) antennas heights [9].

According to the example of Fig. 1, the ray-tracing algorithm applied on quasi three-dimensional indoor environments (RTQ3D-indoor) converts a given path initially obtained from the RT 2D algorithm in five rays: i) the direct path (from the viewpoint of a vertical plane); ii) the ground reflected path; iii) the ceiling reflected path; iv) the path reflected on the ground and on the ceiling and; v) the path reflected firstly on the ceiling and then on the ground [9].

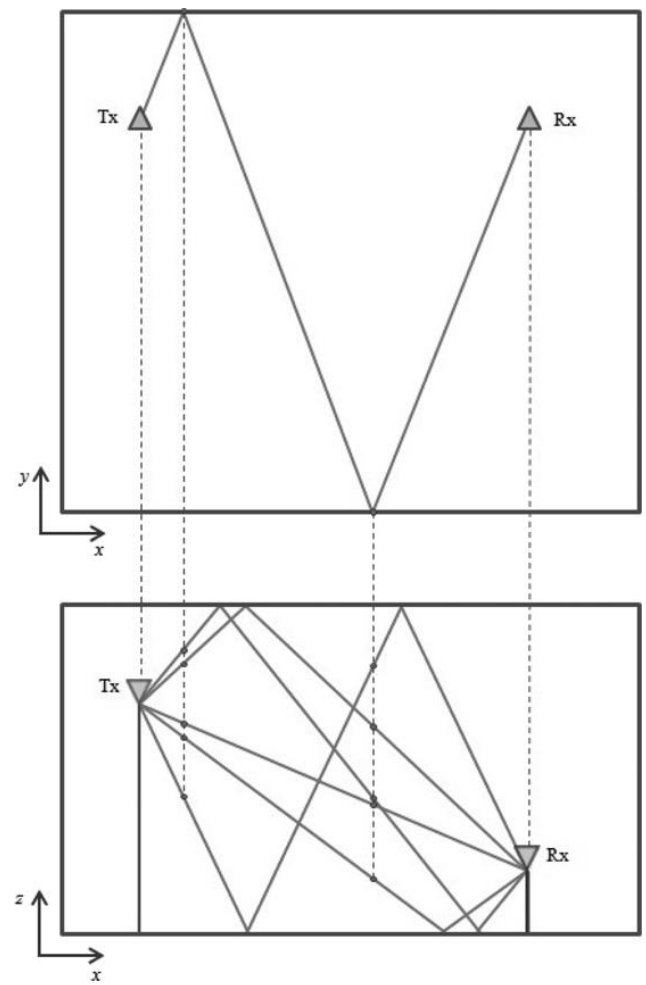

Fig. 1. Passage of a 2D path to five quasi-3D paths using the RTQ3D-indoor algorithm [8]: a) horizontal plane; b) vertical plane [9].

\section{Metamodel: ANN (Artificial Neural Network) with backpropagation algorithm}

According to [10], a neural network is a massively parallel distributed processor consisting of simple processing units, which have the natural tendency for storing experiential knowledge and making it available for use. It resembles the brain in two respects:

- knowledge is acquired by the network from its environment through a learning process;

- connection strengths between neurons, known as synaptic weights, are used to store the acquired knowledge.

The procedure used to perform the learning process is called a learning algorithm, whose function is 
to modify the synaptic weights of the network in a systematic manner to achieve a desired design goal. We consider the multilayer perceptron (MLP), which consists in a network of simple neurons called perceptrons. The MLP is a hierarchical structure of several perceptrons with some advantages compared to single-layer networks and it is capable of learning a rich variety of nonlinear decision surfaces [10-11].

The so-called neuron's net input can be represented by the weighted sum of the inputs from other neurons and external inputs, according to (3).

$$
n e t_{i}(t)=\sum_{j=1}^{p} w_{j i} y_{j}(t-1)+\sum_{l=1}^{m} w_{k i} I_{k}(t)+\theta_{i}
$$

Where $y_{j}(t-1)$ is the output of the $\mathrm{j}$-th neuron in the previous processing cycle $(\mathrm{t}-1) ; I_{k}$ is the $\mathrm{k}$-th external input; $\theta_{i}$ is the bias of the $\mathrm{i}$-th neuron and $w$ refers to the corresponding weight, shown in Fig.2[10].

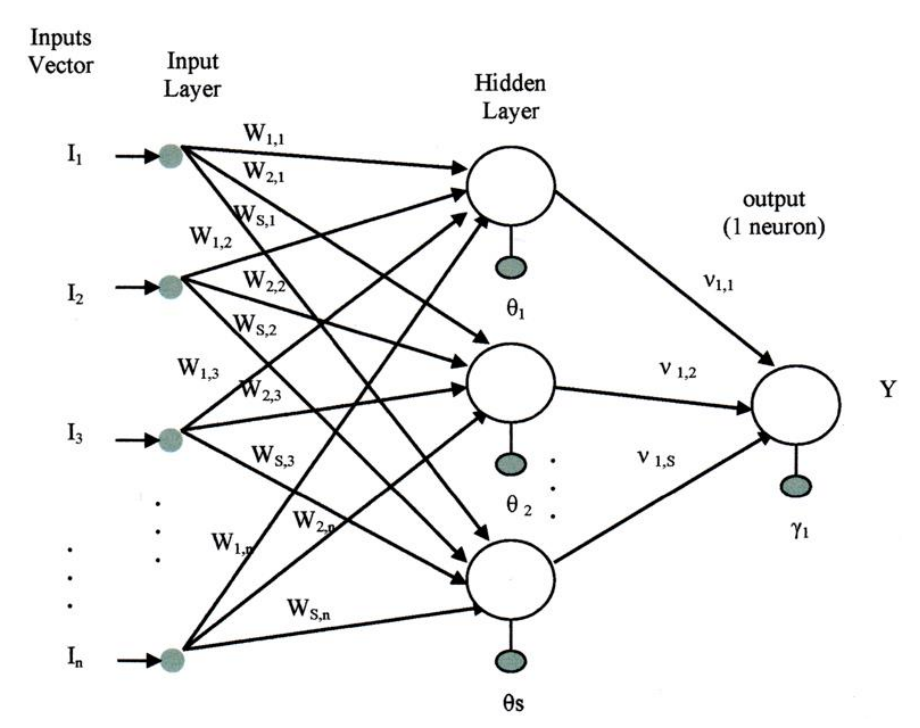

Fig.2. Illustrative "Graph" of The proposed network [10].

In order to model an electromagnetic problem, data from the electromagnetic simulation are used to train the ANN, i.e., to compute the weights of (3). Here, we chose to use the backpropagation (BP) algorithm for this training (supervised learning). Backpropagation is an abbreviation for "backward propagation of errors". In the BP algorithm, the network begins with a random set of weights. Input data is normalized to a range of \pm 1 and input vectors are presented to the network. Thus, the corresponding output is calculated using the initial weight matrix. Next, the ANN output is compared to the output of the electromagnetic simulation (using the same input data). The squared difference between the two output vectors determines the system error. The accumulated error for all of the input-output pairs is defined as the Euclidean distance in the weight space which the network attempts to minimize. Minimization is accomplished via a gradient descent approach, in which the weights are adjusted in the direction of decreasing error. When the error has been decreased below a pre-defined tolerance, training ceases. It is worth remarking the nonlinear functions can be represented by MLP 
with units that use nonlinear activation functions. Since the BP algorithm requires an activation function to be differentiable, we used a sigmoid (logistic function) [10]. Another important aspect in defining the topology of neural network is the number of neurons in the hidden layer, due to its influence on both the speed and the effectiveness of the learning network, which was defined by the geometric mean between the input and output neurons [11].

\section{VALIDATION}

In order to verify the effectiveness of proposed method, it was initially used in the optimization of a test function with analytical solution. Specifically, the goal is to find the global maximum of the Peaks function, given by (4) and shown in Fig. 3. It is important to mention that the Rastrigin and Sinc functions were also tested during the validation process of this study, with satisfactory and similar results for all of them. The results obtained through the functions Peaks, Rastring and Sinc showed the efficiency and effectiveness necessary to validate the proposed PSO optimizer assisted by an ANN.

$$
F(x, y)=3(1-x)^{2} e^{-x^{2}-(y+1)^{2}}-\left(2 x-10 x^{3}-10 y^{5}\right) e^{-x^{2}-y^{2}}-\frac{1}{3} e^{-(x+1)^{2}-y^{2}}
$$

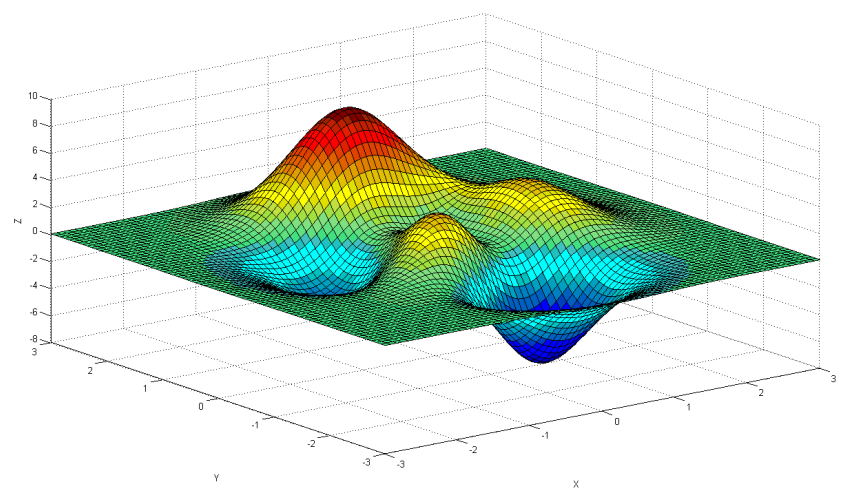

Fig.3. The analytical function considered.

The goal of this implementation is to test the effectiveness of the PSO optimizer using an ANN (network MLP with backpropagation algorithm) as a metamodel.

The optimization was done both directly using the function given by (4) and using an ANN-based metamodel to represent it. Specifically, the PSO was used to obtain the global maximum location of Peaks function. The algorithm was initialized with a random set of particles and the process took place until the maximum point was located, at the center of the red contours.

Fig. 4(a) shows the contour plot of the results obtained using directly (4) while fig. 4(b) shows the results obtained using the ANN simulating the function given by (4). The global maximum is represented by " $\times$ " in fig. 4(a). 


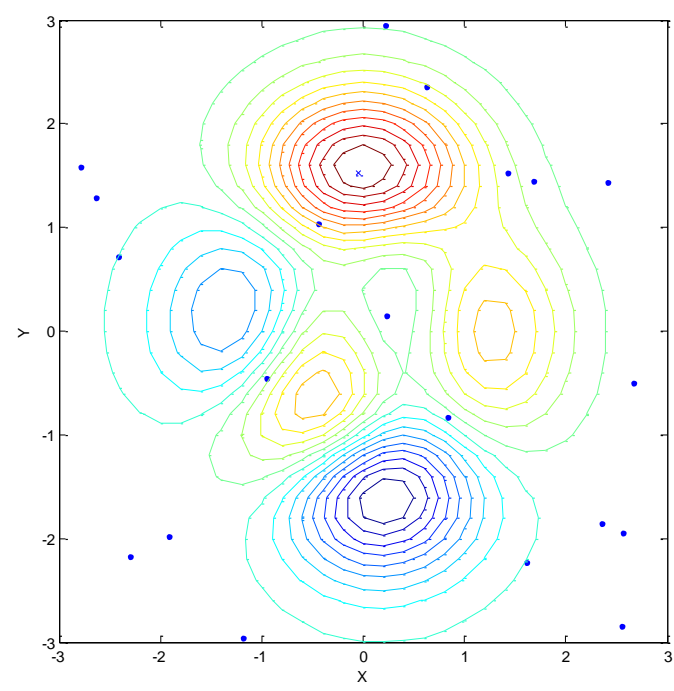

(a)

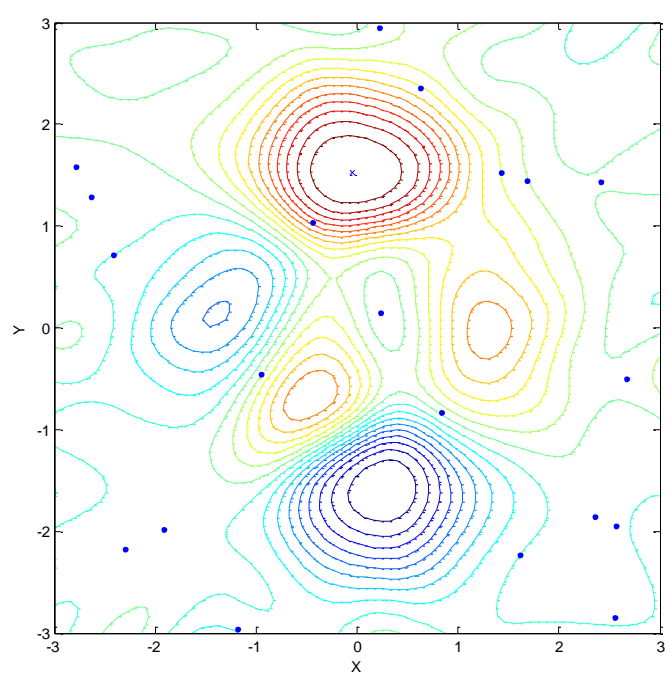

(b)

Fig.4. (a). Contour plot of the actual function; (b) Contour plot of the simulated function, with the located peak.

The ANN was trained until a root mean square error of 0.0004 was obtained, requiring 27,253 epochs to get this error value. An epoch is a step in the training process of an ANN, i.e., each time the network is presented with a new input pattern. The root mean square error solution of PSO assisted by the ANN was equal to 0.00569. From the results, we can conclude that the use of the proposed metamodel to replace the original fitness function allows obtaining good results.

\section{ELECTROMAGNETIC PROBLEM}

The use of an electromagnetic prediction model represents an important design procedure in wireless systems. Here, we use a quasi-3D ray-tracing propagation model associated with a PSO optimizer to find optimal antenna placement in an indoor scenario [9-11].

Initially, a population is generated where each particle corresponds to the coordinates $(\mathrm{x}, \mathrm{y})$ of the 
transmitting antennas placements in a $40 \mathrm{~m} \times 28 \mathrm{~m}$ scenario. It was defined a mesh of 160 receptors uniformly distributed within the environment. The antennas of all receivers were considered to be 1.5 $\mathrm{m}$ high from the ground. The method was applied to minimize the number of points where the field is below a minimum reception threshold $(-110 \mathrm{dBm})$. This is equivalent to maximize the coverage area. The variable of the problem was assumed to be a vector containing the coordinates $(x, y)$ of the transmitting antennas, allowing different locations of them on the horizontal plane of the scenario. The height of these transmitting antennas was assumed to be fixed $(3 \mathrm{~m})$, simulating vertically polarized quarter-wave monopole antennas fixed to the ceiling. Two antennas were considered in the problem. Therefore, the variable of the problem is a vector containing four elements, which represent coordinates of transmitting antenna positions on the horizontal plane. The frequency used was 2.4 $\mathrm{GHz}$, and the total equivalent isotropically radiated power (EIRP) was $400 \mathrm{~mW}$.

Firstly, the field values generated by ray-tracing are used as a guide in the learning process performed by the network, optimizing weight values, which are saved in a file. After training, the ANN calculates the field values at all reception points, which are used by the PSO optimizer, allowing a great reduction in computation time.

The PSO assisted by ANN was initialized with a set of 100 random particles. The PSO assisted by ANN optimizing was performed for three different numbers of iterations: 2,000, 3,000 and 6,000. Table I shows the best antenna locations found by the PSO for these three cases. We observe that the results are very sensitive to the number of iterations, which indicates that the ANN needs to be further improved. Fig. 5 shows the mapping of received powers considering the best pair of positions of the antennas found for 3,000 iterations. We see that the distribution of field is reasonable. Best results, with more uniform fields, can be obtained using more transmitting antennas in the scenario.

TABLE I. RESULTS OF OPTIMIZATION ANN-ASSISTED PSO

\begin{tabular}{ccc}
\hline Iterations & $\begin{array}{c}\text { Positions Antenna 1 } \\
\left(\mathbf{x}_{\mathbf{1}}(\mathbf{m}), \mathbf{y}_{\mathbf{1}}(\mathbf{m})\right)\end{array}$ & $\begin{array}{c}\text { Positions Antenna 2 } \\
\left(\mathbf{x}_{\mathbf{2}}(\mathbf{m}), \mathbf{y}_{\mathbf{2}}(\mathbf{m})\right)\end{array}$ \\
\hline 2,000 & $(25.61,9.56)$ & $(34.04,17.58)$ \\
3,000 & $(20.18,7.56)$ & $(29.12,15.82)$ \\
6,000 & $(34.50,4.91)$ & $(32.00,6.65)$ \\
\hline
\end{tabular}




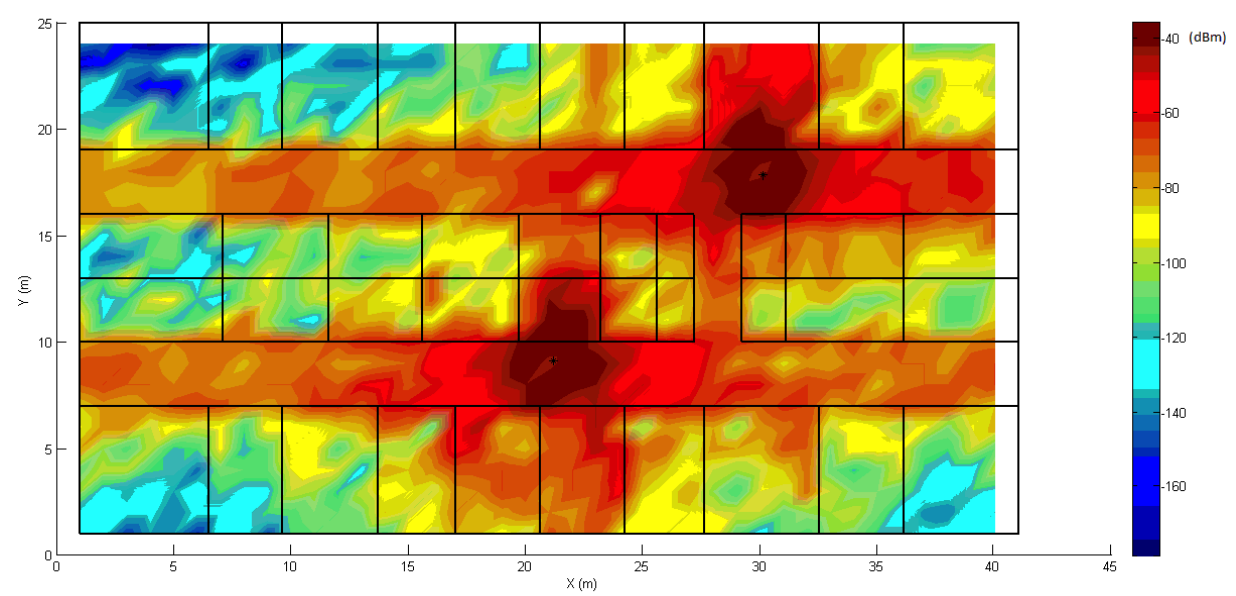

Fig.5. Received power mapping in the indoor environment (3,000 iteractions).

\section{CONCLUSIONS}

In this paper, we proposed a PSO optimizer assisted by an ANN, the optimizer was implemented using the MATLAB program version 7.10.0.499. The optimization process was carried out through a MacBook Pro Retina Computer with 2,6 GHz intel Core i5 processor and 8GB of RAM memory $1600 \mathrm{MHz}$ DDR3, with $256 \mathrm{~GB}$ of flash storage. In the optimization of an electromagnetic problem, the computational cost can be very large, since it is necessary to carry out a numerical simulation for each evaluation of the fitness function. In this case, the use of an ANN as a metamodel permits to obtain satisfactory results with a reduction of the computational cost. The study developed in this work is based on [9], where the association of RTQ3D-indoor with PSO optimizers was applied in a typical telecommunication problem: a wireless local area network (WLAN) system in an indoor scenario that must be covered with two $802.11 \mathrm{~b} / \mathrm{g} / \mathrm{n}(2.4 \mathrm{GHz})$ access points (AP). However, the study developed in [9] disregards the computational cost, which is quite high when a metamodel is not used. As an example, the average time required for optimization using the ANN metamodel with 3,000 epochs is around $36 \mathrm{~min}$. Without using the metamodel, the PSO takes about 72 hours to produce slightly better results.

In order to assess the effectiveness of the proposed method, it was applied in a test function with a global maximum. Also, it was applied in an electromagnetic problem (namely, the best antenna positioning in wireless systems). The response of the ANN-assisted PSO for a real electromagnetic problem can be considered satisfactory. All observations suggest that an improvement in the ANN and additional training will lead to the best results. However, this would increase the computational time. 
Journal of Microwaves, Optoelectronics and Electromagnetic Applications, Vol. 15, No. 4, December 2016427 DOI: http://dx.doi.org/10.1590/2179-10742016v15i4816

\section{REFERENCES}

[1] H.P. Schwefel, E. L. Taylor, "Evolution and Optimum Seeking," John Wiley \& Sons. Inc, United States of America, pp. 87-88, 1994.

[2] G. Venter, and J. Sobieszczanski-sobieski, "Particle Swarm Optimization," Proceedings of the 43rd AIAA/ASME/ASCE/AHS/ASC Strutures, Structural Dynamics, and Materials Conference, Denver, CO, Vol. AIAA-2002-1235, April 22-25 2002.

[3] L. Lebensztjan, C. A. R. Marretto, M. C. Costa, J. L. Coulomb, "Kriging: a useful tool for electromagnetic device optimization," vol.40, N'.2. IEEE Transactions on Magnetics, March 2004, p. 1196-99.

[4] M. T. M. Emmerich, C. M. Varcol, "Metamodel-Assisted Evolution Strategies in Electromagnetic Compatibility Design," EUROGEN. 1-12, 2005.

[5] L. Willmes, T. Bäck, Y. Jin, "Sendhoff, B. Comparing neural network and kriging for fitness approximation in evolutionary optimization," IEEE, 2003 pp. 670

[6] Y. Rahmat-Samii, N. Jin, "Particle Swarm Optimization (PSO) in Engineering Electromagnetics: A Nature-Inspired Evolutionary Algorithm," IEEE, 2007. Inc, United States of America, 1994 pp. 87-88.

[7] R. C. Eberhart and Y. Shi, "Particle Swarm Optimization: Developments, Applications and Resources," Congress on Evolutionary Computation, 2001, vol. 1, pp. 81-86, 2001.

[8] S. Grubisic, W. P. Carpes Jr, J. P. A. Bastos, "An Efficient indoor ray- tracing propagation model with a quasi-3D approach," IEEE CEFC 2012, November 2012, Oita/Japan.

[9] S. Grubisic, W. P. Carpes Jr, J. P. A. Bastos, G. Santos, "Association of a PSO optimizer with a quasi-3D ray-tracing propagation model for mono and multi-criterion antenna positioning in indoor environments," IEEE Transactions on Magnetics, Vol. 49, No. 5, May 2013.

[10] S. Haykin, "Neural Networks: A Comprehensive Foundation," Prentice Hall, 1999

[11] J. M. Barreto, "Neural Networks: Mathematical and Computational Aspects," Annals of the Brazilian Society of Applied and Computational Mathematics (BSACM), 1996. 